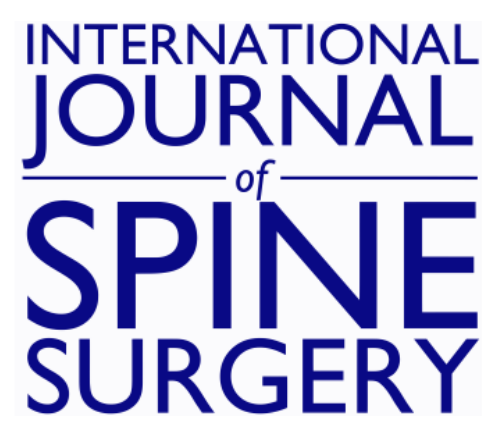

\title{
Modic Changes on Magnetic Resonance Imaging and Smoking History Predict Vascular Adherence During Anterior Lumbar Exposure
}

Gregory M. Malham, Nicholas Johnson and Matthew H. Claydon

Int J Spine Surg 2021, 15 (6) 1054-1059

doi: https://doi.org/10.14444/8190

http://ijssurgery.com/content/15/6/1054

This information is current as of April 26, 2023.

Email Alerts Receive free email-alerts when new articles cite this article. Sign up at:

http://ijssurgery.com/alerts

The International Journal of Spine Surgery

2397 Waterbury Circle, Suite 1,

Aurora, IL 60504, Phone: +1-630-375-1432 


\title{
Modic Changes on Magnetic Resonance Imaging and Smoking History Predict Vascular Adherence During Anterior Lumbar Exposure
}

\author{
GREGORY M. MALHAM, MBChB, FRACS ${ }^{1}$; NICHOLAS JOHNSON, MBBS ${ }^{2}$; AND MATTHEW H. CLAYDON, \\ MBBS, BMedScI, FRACS ${ }^{2,3}$ \\ ${ }^{I}$ Neuroscience Institute, Epworth Hospital, Melbourne, Australia; ${ }^{2}$ Department of Vascular Surgery, The Alfred, Melbourne, Australia, ${ }^{3}$ Department of Vascular \\ Surgery, Epworth Hospital, Melbourne, Australia
}

\begin{abstract}
Background: Anterior approaches to surgically access the lumbar intervertebral discs are associated with a risk of developing major vascular injury and bleeding. The likelihood of injury increases in the presence of dense adherence between the disc annulus, vertebral periosteum, and vessels, which result from reactive inflammatory changes that cause fibrous scarring.

Objective: To identify factors that predict vascular adherence, which may facilitate preoperative planning and technique modifications for anterior spine surgery.

Study Design: Prospective study examining patients undergoing anterior retroperitoneal exposure for lumbar disc surgery.

Methods: A total of 246 consecutive patients were enrolled in this study, all of whom had anterior retroperitoneal exposure for lumbar disc surgery. Patient demographics, smoking status, magnetic resonance imaging (MRI) findings, operative parameters, and dissection difficulty associated with vascular adherence were recorded. Current smokers were defined as those who smoked at the time of surgery or had ceased smoking $<6$ months before the operation. Patients were excluded if they were morbidly obese or had previously undergone anterior spine surgery or radiotherapy.

Results: A multivariate regression analysis identified 2 significant risk factors for difficult dissections that are complicated by vascular adhesion: Modic Type 2 changes on MRI $(P=0.009)$ and any history of smoking $(P=0.007)$. Patients with Modic Type 2 changes or a smoking history were 2.1 and 2.2 times more likely to present with vascular adherence, respectively.

Conclusions: Modic Type 2 changes on MRI and any smoking history can predict the adherence of large blood vessels to the anterior disc annulus, which enhances the difficulty of the dissection. These predictors could indicate to spinal surgeons that the patient has a 2-fold increased risk of vascular adherence.

Clinical Relevance: Modic Type 2 changes on MRI and any smoking history can predict the adherence of large blood vessels to the anterior disc annulus, which enhances the difficulty of the dissection. These predictors could indicate to spinal surgeons that the patient has a 2-fold increased risk of vascular adherence during anterior lumbar exposure.
\end{abstract}

Level of Evidence: 3.

Lumbar Spine

Keywords: anterior spine surgery, lumbar disc surgery, anterior lumbar interbody fusion, total disc replacement, anterior retroperitoneal exposure, magnetic resonance imaging, Modic changes, smoking, vascular adherence, vascular injury

\section{INTRODUCTION}

The anterior approach to lumbar disc surgery, for anterior lumbar interbody fusion (ALIF) ${ }^{1,2}$ or total disc replacement (TDR), ${ }^{3,4}$ is frequently applied, despite the inherent risks of developing major vascular injury and significant bleeding events. ${ }^{5,6}$ The vascular injury rate associated with anterior exposure ranges from $1.9 \%$ to $6.1 \%$. $^{7,8}$ Garg et al identified that the likelihood of experiencing vascular injury and increased blood loss was greater in males, patients with 2-level exposures, and those with a high body mass index (BMI). The most commonly injured vessels are the left common iliac vein, the inferior vena cava, and the ascending iliolumbar vein. ${ }^{9}$ Veins are more commonly injured than other blood vessels ${ }^{8}$ because of the close association between these structures and the target discs. A paucity of studies exist that explore the direct pathophysiology of these injuries. Moreover, reactive inflammatory changes can lead to the formation of fibrous scarring, which can form dense adherences between the disc annulus, vertebral periosteum, and blood vessels. ${ }^{6}$ We hypothesized that the formation of these adherences may represent a correlation between anterior surgical approaches and vascular injuries. To date, no published studies have identified the factors that predict the formation of these adhesions. In this study, we aimed to identify 
predictive factors in patients to identify vascular adherence to facilitate preoperative planning and technique modifications when performing anterior spine surgery.

\section{MATERIALS AND METHODS}

We conducted a prospective study, which included consecutive patients who underwent anterior spine surgery between 2011 and 2018 performed by a single spine surgeon (G.M.M.) and a single vascular surgeon (M.H.C.).

\section{Inclusion/Exclusion Criteria}

Indications for surgery included severe discogenic pain, radiculopathy, and Grade 1 or 2 degenerative or isthmic spondylolisthesis at L4-5 and/or L5-S1. Exclusion criteria included disease progression more severe than a 2-level disc disease, Grade 3 or 4 spondylolisthesis, significant iliac artery disease (ie, heavy calcification, aneurysmal, and/or severe stenosing atherosclerotic disease), morbid obesity (BMI $\geq 35$ ), and previous complex/extensive retroperitoneal surgery or abdominal/pelvic radiotherapy.

\section{Patient Characteristics}

We collected patient data, including demographics, smoking status (current smokers were defined as patients who smoked at the time of surgery or had ceased smoking $<6$ months preoperatively), a previous history of spine operation, magnetic resonance imaging (MRI) findings, and operative parameters, including the level(s) of the operation and the difficulty of dissection due to vascular adherence.

\section{Modic Changes}

MRI signal intensity changes observed in the vertebral body marrow adjacent to endplates were classified into Modic Types 1, 2, and 3. ${ }^{10}$ Modic Type 1 changes describe a decreased signal intensity on T1-weighted images and an increased signal intensity on T2-weighted images; Modic Type 2 changes describe an increased signal intensity on both T1 and T2 images; and Modic Type 3 changes describe a decreased signal intensity on both $\mathrm{T} 1$ and $\mathrm{T} 2$ images. Two independent radiologists from the treating institution evaluated the MRI scans.

\section{Surgical Techniques}

The surgical techniques used for $\mathrm{ALIF}^{9}$ and $\mathrm{TDR}^{11}$ have been previously described elsewhere. A hybrid procedure can also be performed, consisting of ALIF at the L5-S1 level and TDR at the L4-5 level. The vascular surgeon performed a right-lower Rutherford-Morrison

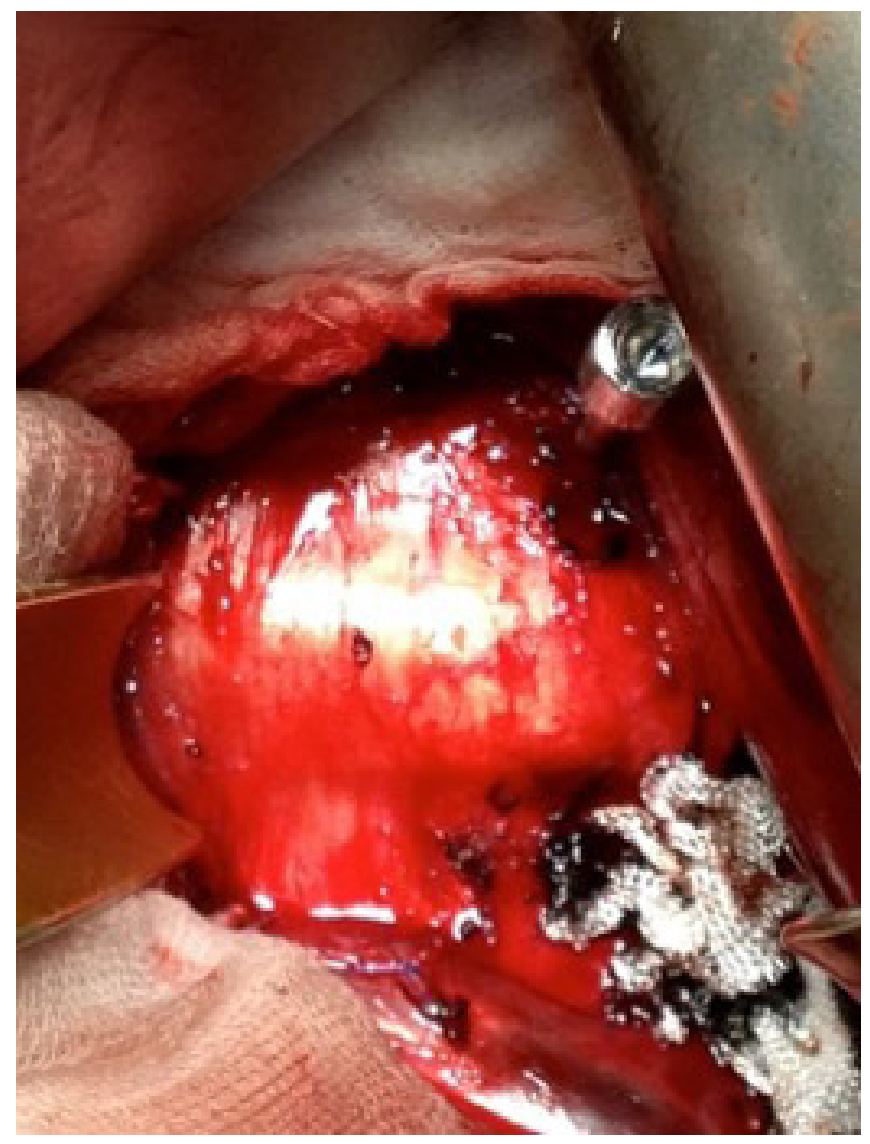

Figure 1. Intraoperative photograph showing a standard vascular dissection between a vein (secured with vascular retraction pins) and the smooth periosteum to expose the L5-S1 disc with a midline pin.

transverse incision using a retroperitoneal approach to access the L5-S1 level. For the L4-5 level and 2-level exposures, the vascular surgeon performed a midline lower abdominal incision using a left-sided retroperitoneal approach.

\section{Difficulty of Dissection Due to Vascular Adherence}

The vascular access surgeon was blinded to the patients' smoking status and Modic changes on MRI. A binary, subjective evaluation of the degree of adherence was performed by the vascular surgeon, who classified each dissection as either standard (Figure 1) or difficult (Figure 2), depending on the forces and techniques required for vessel mobilization. A difficult dissection was defined by (1) the presence of broad vessel adherence, particularly to the left common iliac vein, the anterior vertebral body, and the disc, which was not attributable to perforating vessels and required increased dissection to mobilize the vessels; and (2) the necessity of using a subperiosteal approach to achieve safe separation and vessel mobilization. 


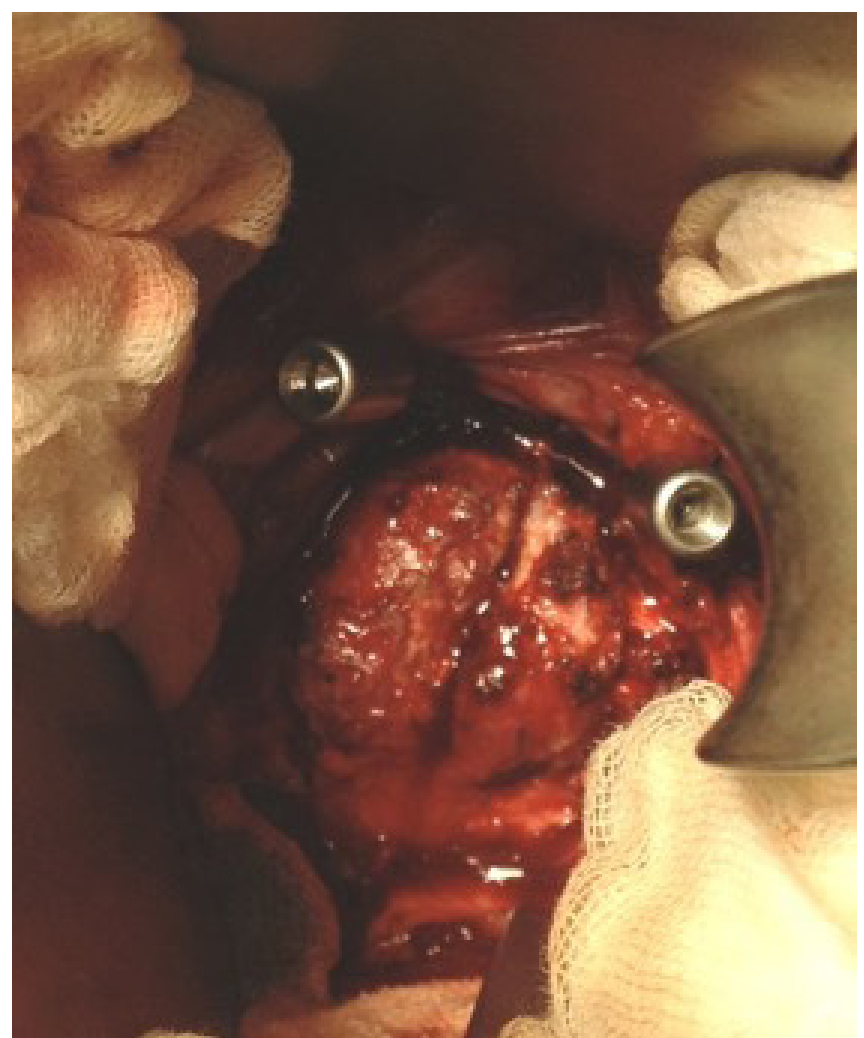

Figure 2. Intraoperative photograph demonstrating a difficult vascular dissection that features thickened, "gritty" inflammation and the loss of the usual periosteal sheen, requiring a subperiosteal surgical approach to safely expose the L5-S1 disc.

\section{Vascular Injury}

Significant vascular injury was defined as the loss of $>150 \mathrm{~mL}$ blood from a single major vessel or any injury to a major vessel that required suture repair. Blood loss was measured by a Cell Saver Autologous Blood Recovery System (Haemonetics Corp., Braintree, MA, USA), which was operated by a certified perfusionist.

\section{Statistical Analysis}

Multivariate regression analysis was conducted using SPSS software (Version 21.0, IBM Corp., Armonk, NY, USA). Statistical significance was set to $P<0.05$.

\section{RESULTS}

\section{Patient Characteristics}

The patient demographics and treatment information are provided in Table 1. In this series, $17.5 \%$ (43 of 246) of patients were classified as having difficult dissections due to vascular adherence.
Table 1. Patient demographic and treatment information $(\mathrm{N}=246)$.

\begin{tabular}{|c|c|}
\hline Characteristic & Values \\
\hline Age in years, mean ( $\mathrm{SD}$, range) & $42.5(11.9,21-81)$ \\
\hline Male, n $(\%)$ & $124(50)$ \\
\hline BMI, mean (SD, range) & $26.2(3.8,19-37)$ \\
\hline \multicolumn{2}{|l|}{ Comorbidities, $\mathrm{n}(\%)$} \\
\hline Ex-smoker & $83(34)$ \\
\hline Current smoker & $38(15)$ \\
\hline Never smoker & $125(51)$ \\
\hline Prior microdiscectomy, n (\%) & $17(7)$ \\
\hline \multicolumn{2}{|c|}{$\begin{array}{l}\text { Vascular adherence/dissection difficulty, } \\
\mathrm{n}(\%)\end{array}$} \\
\hline Standard & $203(82)$ \\
\hline Difficult & $43(18)$ \\
\hline \multicolumn{2}{|l|}{ Blood loss (mL) } \\
\hline \multicolumn{2}{|l|}{ Standard dissection } \\
\hline Single-level mean (SD, range) & $126.6(116.8,20-490)$ \\
\hline Two-level mean (SD, range) & $188.9(202.6,50-650)$ \\
\hline \multicolumn{2}{|l|}{ Difficult dissection } \\
\hline Single-level mean (SD, range) & $160.3(280.7,10-1420)$ \\
\hline Two-level mean (SD, range) & $347.5(355.3,20-1550)$ \\
\hline \multicolumn{2}{|l|}{ Type of operation, $\mathrm{n}(\%)$} \\
\hline ALIF & $117(48)$ \\
\hline TDR & $69(28)$ \\
\hline Hybrid & $60(24)$ \\
\hline \multicolumn{2}{|l|}{ Level of operation, $\mathrm{n}(\%)$} \\
\hline \multicolumn{2}{|l|}{ Single-level } \\
\hline L4-5 & $50(20)$ \\
\hline L5-S1 & $121(49)$ \\
\hline \multicolumn{2}{|l|}{ Two-level } \\
\hline L3-4 and L4-5 & $2(1)$ \\
\hline L4-5 and L5-S1 & $73(30)$ \\
\hline \multicolumn{2}{|l|}{ Modic changes, $\mathrm{n}(\%)$} \\
\hline Type 1 & $11(4)$ \\
\hline Type 2 & $57(23)$ \\
\hline
\end{tabular}

Abbreviations: ALIF, anterior lumbar interbody fusion; BMI, body mass index; TDR, total disc replacement.

\section{Factors Predicting Difficult Dissection}

Multivariate regression analysis was performed to identify factors that predict a difficult dissection due to vascular adherence, which identified 2 significant risk factors: Modic Type 2 changes on MRI and smoking status (Table 2).

Among patients with Modic Type 2 changes at the operative level, 16 of 57 patients $(28 \%)$ were classified as difficult dissections, whereas only 25 of 189 patients (13\%) without Type 2 changes were classified as difficult dissections $(P=0.014)$. Thus, patients with Modic Type 2 changes were 2.1 -fold more likely to

Table 2. Multiple regression analysis of a difficult dissection.

\begin{tabular}{ll}
\hline Factor & $\boldsymbol{P}$ Value \\
\hline Age & 0.0981 \\
Sex & 0.7377 \\
BMI & 0.2497 \\
Smoking status & 0.0072 \\
Prior microdiscectomy & 0.9009 \\
Level & 0.9737 \\
Modic Type 1 changes & 0.1266 \\
Modic Type 2 changes & 0.0085 \\
\hline
\end{tabular}

Abbreviation: BMI, body mass index. 
present with difficult dissection conditions than patients without Type 2 changes.

In addition, 9 of 38 patients (24\%) who were current smokers and 19 of 83 patients (23\%) who were exsmokers were classified as difficult dissections, whereas only 13 of 125 patients (10\%) who never smoked presented with a difficult dissection $(P=0.01)$. Thus, patients with any smoking history were 2.2 times more likely to present with difficult dissection conditions than nonsmoking patients.

\section{Vascular Injury}

No significant vessel injuries occurred during this series. The main source of intraoperative blood loss was from epidural and median sacral vessels with the lumbosacral dissection. When the dissection was difficult, an increase in the measured blood loss occurred during both single-level and 2-level exposures. The average blood loss was $126.6 \mathrm{~mL}$ (standard dissection) vs 160.3 $\mathrm{mL}$ (difficult dissection, $P=0.5$ ) for single-level exposures, and $189.9 \mathrm{~mL}$ (standard dissection) vs $347.5 \mathrm{~mL}$ (difficult dissection, $P=0.1$ ) for 2-level exposures. The relationship between blood loss and dissection difficulty was not significant.

\section{DISCUSSION}

We have identified 2 independent factors that significantly correlate with the likelihood of dense adhesions between the disc annulus, vertebral periosteum, and adjacent vessels. The ability to predict such adherence, with the associated increased risk of major intraoperative hemorrhage would improve patient consent for surgery. This would allow improved preoperative planning, recruitment of vascular access surgeons, and the provision of equipment such as a Cell Saver. The consideration of alternative surgical approaches may be warranted, especially in patients who already have other relative contraindications such as obesity, concomitant arterial pathology (calcified atherosclerosis, tortuosity, or ectasia), or multiple previous abdominal operations.

In our study, a standard dissection was possible when the anterior surfaces of the vertebral bodies and discs were smooth, with a low sheen. Although occasional adherence focal points can be observed between the vessels and the vertebral bodies, particularly where the perforating vessels enter and exit the bone, the vessels, including the left common iliac vein that is in direct apposition to the vertebral bodies, could be easily separated from the periosteum and the surface of the disc with the application of peanut sponge forceps. In contrast, dissections were considered difficult when generalized adherence between these vessels, the vertebral body, and the disc were observed. This resulted in the veins being abnormally resistant to separation from these surfaces and required an increased dissection, which also increased the risk of venous injury. The loss of the characteristic sheen was also observed, with the final exposed surface appearing "gritty." A continued effort to separate the vessels from the periosteum yielded an unacceptably high risk of developing major vascular injury during difficult dissections. To achieve a safe separation of the vessels from the target disc level, a deeper, subperiosteal approach must be used to develop a plane between the bone and periosteum, which allows adherent vessels to remain attached to the periosteum. This aforementioned approach is more appealing than that of a more superficial plane between the periosteum and the vein, which is more difficult and time-consuming.

Blood loss increased during difficult dissections due to adherence compared with standard dissections in both single-level and 2-level exposures. Notably, this increase was not significant. The vascular access surgeon employed advanced surgical techniques during difficult dissections to protect and mobilize, thus allowing for the safe exposure of the target disc. Had these advanced techniques not been employed, the continued efforts to separate the periosteum from the veins would likely have resulted in major vascular injury, and the consequent increase in blood loss would have been significant.

Michael Modic, at the Cleveland Clinic, described changes in MRI signal intensity, observed in the vertebral body marrow adjacent to endplates, as Type 1 , 2 , or 3 changes. ${ }^{11,12}$ Type 1 changes occur in approximately $4 \%$ of patients undergoing MRI for disc disease and represent acute inflammatory changes (eg, edema, hyperemia, and leucocyte infiltration) in the subchondral bone marrow. Type 2 changes occur in approximately $16 \%$ of patients and represent lipid replacement in the subchondral marrow. Type 3 changes are the least common $(<1 \%$ of patients), and represent extensive bony sclerosis with little residual marrow. ${ }^{13}$ Type 1 but not Type 2 changes have been associated with increased lower back pain and disability, ${ }^{14}$ and Type I changes can progress to Type 2 changes over time. ${ }^{15}$ Modic changes are most commonly observed at L4-5 and L5-S1 and usually occur anteriorly. ${ }^{12}$

Although previous reports have correlated Modic changes with the pathology of the vertebral body marrow, no reports have associated Modic changes with perivertebral 
inflammation, scarring, or vessel adherence. In this study, we identified an association between intravertebral body Modic Type 2 changes, extravertebral inflammation, and scarring. The presence of Modic Type 2 changes suggested the chronicity of degenerative disc disease, which causes histopathological changes in the bone marrow. These Type 2 changes reflect chronic inflammation, which may result in dense scarring. This progression also appears to occur perivertebrally and causes scarring, and doubles the risk of vascular adherence.

We also found an association between vascular adherence and any history of smoking, even in the absence of Modic changes. This is possibly because smoking causes a chronic, low-grade, systemic inflammatory response that is associated with elevated levels of white blood cells and inflammatory mediators, such as C-reactive protein, fibrinogen, interleukin- $6,{ }^{16}$ and tumor necrosis factor- $\alpha .{ }^{17}$ Cigarette smoke-induced inflammation affects the vascular endothelium and makes it more permeable to inflammatory cells that lead to damage caused by reactive oxygen species. ${ }^{18,19}$ Smoking also lowers the protective anti-inflammatory levels of nitric oxide in the endothelium. ${ }^{20}$ The resultant perivascular changes, combined with degenerative disc disease, can induce chronic inflammation, scarring, and vascular adherence.

Requiring patients to cease smoking before surgery is necessary for several reasons. Postoperative respiratory complications are reduced in patients who cease to smoke $\geq 4$ weeks before surgical procedures. ${ }^{21}$ An increased benefit is observed if the cessation of smoking occurs $\geq 8$ weeks before surgery. ${ }^{22,23}$ Postoperative wound-healing complications are also reduced in patients who cease to smoke $>3$ to 4 weeks prior to surgery. ${ }^{21,24}$ Because the greatest benefits associated with smoking cessation occur after cessation periods longer than 6 months, ${ }^{25}$ we defined ex-smokers as those who ceased to smoke $>6$ months preoperatively. Such a time period seemingly permitted for the smoking-induced inflammation and the resulting vascular adherence to subside. Therefore, any inflammation or vascular adherence encountered intraoperatively could reasonably be attributed to Modic changes alone. However, the effects of smoking are enduring, and even long durations of smoking abstinence are associated with an increased risk of developing vascular adherence. C-reactive protein levels remain significantly elevated in ex-smokers, from a period up to 10 to 20 years after smoking cessation. This suggests the persistence of a low-grade inflammatory response in former smokers. ${ }^{16}$ Consistent with this finding, we found that any smoking history doubled the risk of developing vascular adherence.
Ultimately, the causes of inflammation and vascular adherence may be multifactorial, ${ }^{18,19}$ as reflected by the increased adherence observed in patients in our series with a history of smoking but without Modic changes. The predominant inciting factor in some patients may be associated with vertebral inflammatory changes, as indicated by Modic changes, whereas perivascular inflammation may occur in other patients due to their smoking history. Disc pathology did not appear to be a major inciting factor.

For surgeons who are inexperienced in anterior spine exposure and do not utilize the services of a spinal access surgeon, the presence of Modic Type 2 changes, especially when combined with any smoking history, represents a valuable preoperative warning that indicates that a more experienced surgeon may be necessary. Similarly, in patients with various contraindications, the presence of Modic Type 2 changes and any smoking history may indicate that an alternative approach should be used. Although rare, major vascular injury can be fatal or threaten limb viability. Vascular adherence increases the risks of such an event occurring. This study illustrates 2 valuable preoperative measures that can be assessed to predict vascular adhesions that may lead to catastrophic vascular injury.

The strengths of this study include the use of a consistent surgical technique, performed by a single spine surgeon and a single vascular surgeon. The vascular surgeon was blinded to the patients' smoking status and Modic changes on MRI to eliminate bias on the degree of operative vessel adherence. Two independent radiologists from the treating institution evaluated all MRI scans. A Cell Saver, operated by a certified perfusionist, measured blood loss.

However, the limitations of this study include a relatively small sample size compared with those featured in multicenter studies. In addition, another limitation is the lack of histopathological confirmations regarding the underlying causes of vascular adherence, given that no tissue was sampled for examination. One potential issue is that a vascular surgeon's assessment of adherence and dissection difficulty may alter over time. However, the vascular surgeon that participated in our study had extensive prior experience with vascular exposure and is beyond the learning curve. ${ }^{9}$ At our institution, Modic Type 3 changes are not routinely reported; thus, we did not include these changes in our study.

\section{CONCLUSION}

In conclusion, Modic Type 2 changes on MRI and any smoking history predicted the adherence of large vessels to the anterior disc annulus, which enhanced the difficulty of the dissection. These predictors could alert a surgeon, undertaking an anterior exposure, that the patient may 
have a 2-fold increased likelihood of presenting with vascular adherence.

\section{REFERENCES}

1. Burkus JK, Transfeldt EE, Kitchel SH, et al. Clinical and radiographic outcomes of anterior lumbar interbody fusion using recombinant human bone morphogenetic protein-2. Spine. 2002;27(21):2396-2408. doi:10.1097/00007632-20021101000015.

2. Tropiano P, Berjano P, Lamartina C, et al. Anterior lumbar surgical approaches and techniques. Eur Spine J. 2016;25(Suppl 4):488-489. doi:10.1007/s00586-016-4864-y.

3. van den Eerenbeemt KD, Ostelo RW, et al. Total disc replacement surgery for symptomatic degenerative lumbar disc disease: a systematic review of the literature. Eur Spine J. 2010;19(8):1262-1280. doi:10.1007/s00586-010-1445-3.

4. Lu S, Sun S, Kong C, et al. Long-term clinical results following Charite III lumbar total disc replacement. Spine J. 2018;18(6):917-925. doi:10.1016/j.spinee.2017.08.252.

5. Sasso RC, Best NM, Mummaneni PV, et al. Analysis of operative complications in a series of 471 anterior lumbar interbody fusion procedures. Spine. 2005;30:670-674. doi:10.1097/01.brs. 0000155423.18218 .75 .

6. Fantini GA, Pappou IP, Girardi FP, et al. Major vascular injury during anterior lumbar spinal surgery: incidence, risk factors, and management. Spine. 2007;32:2751-2758. doi:10.1097/ BRS.0b013e31815a996e.

7. Brau SA, Delamarter RB, Schiffman ML, et al. Vascular injury during anterior lumbar surgery. Spine J. 2004;4(4):409-412. doi:10.1016/j.spinee.2003.12.003.

8. Garg J, Woo K, Hirsch J, et al. Vascular complications of exposure for anterior lumbar interbody fusion. J Vasc Surg. 2010;51(4):946-950. doi:10.1016/j.jvs.2009.11.039.

9. Malham GM, Parker RM, Ellis NJ, et al. Anterior lumbar interbody fusion using recombinant human bone morphogenetic protein-2: a prospective study of complications. J Neurosurg Spine. 2014;21(6):851-860. doi:10.3171/2014.8.SPINE13524.

10. Modic MT, Steinberg PM, Ross JS, et al. Degenerative disk disease: assessment of changes in vertebral body marrow with MR imaging. Radiology. 1988;166(1 Pt 1):193-199. doi:10.1148/radiology.166.1.3336678.

11. Gragnaniello C, Seex KA, Eisermann LG, et al. Early postoperative dislocation of the anterior Maverick lumbar disc prosthesis: report of 2 cases. J Neurosurg Spine. 2013;19(2):191-196. doi: 10.3171/2013.5.SPINE12753.

12. Zhang Y-. H, Zhao C-. Q, Jiang L-. S, et al. Modic changes: a systematic review of the literature. Eur Spine J. 2008;17(10):1289-1299. doi:10.1007/s00586-008-0758-y.

13. Modic MT, Ross JS. Lumbar degenerative disk disease. Radiology. 2007;245(1):43-61. doi:10.1148/radiol.2451051706.

14. Järvinen J, Karppinen J, Niinimäki J, et al. Association between changes in lumbar Modic changes and low back symptoms over a two-year period. BMC Musculoskelet Disord. 2015;16:98. doi:10.1186/s12891-015-0540-3.

15. Mann E, Peterson CK, Hodler J, et al. The evolution of degenerative marrow (Modic) changes in the cervical spine in neck pain patients. Eur Spine J. 2014;23(3):584-589. doi:10.1007/ s00586-013-2882-6.
16. Yanbaeva DG, Dentener MA, Creutzberg EC, et al. Systemic effects of smoking. Chest. 2007;131(5):1557-1566. doi:10.1378/chest.06-2179.

17. Zhang H, Park Y, Wu J, et al. Role of TNF-alpha in vascular dysfunction. Clin Sci. 2009;116(3):219-230. doi:10.1042/ CS20080196.

18. Csordas A, Bernhard D. The biology behind the atherothrombotic effects of cigarette smoke. Nat Rev Cardiol. 2013;10(4):219-230. doi:10.1038/nrcardio.2013.8.

19. Madani A, Alack K, Richter MJ, Krüger K, et al. Immuneregulating effects of exercise on cigarette smoke-induced inflammation. J Inflamm Res. 2018;11:155-167. doi:10.2147/JIR.S141149.

20. Landmesser U, Hornig B, Drexler H. Endothelial function: a critical determinant in atherosclerosis. Circulation. 2004;109(21 Suppl 1):II27-33. doi:10.1161/01. CIR.0000129501.88485.1f.

21. Møller AM, Pedersen T, Villebro N, et al. Effect of smoking on early complications after elective orthopaedic surgery. J Bone Joint Surg Br. 2003;85(2):178-181. doi:10.1302/0301620x.85b2.13717.

22. Warner MA, Divertie MB, Tinker JH. Preoperative cessation of smoking and pulmonary complications in coronary artery bypass patients. Anesthesiology. 1984;60(4):380-383. doi:10.1097/00000542-198404000-00022.

23. Warner MA, Offord KP, Warner ME, et al. Role of preoperative cessation of smoking and other factors in postoperative pulmonary complications: a blinded prospective study of coronary artery bypass patients. Mayo Clin Proc. 1989;64(6):609-616. doi:10.1016/ s0025-6196(12)65337-3.

24. Padubidri AN, Yetman R, Browne E, et al. Complications of postmastectomy breast reconstruction in smokers, ex-smokers, and nonsmokers. Plast Reconstr Surg. 2001;107(2):350-351. doi:10.1097/00006534-200102000-00008.

25. Buist AS, Sexton GJ, Nagy JM, et al. The effect of smoking cessation and modification on lung function. Am Rev Respir Dis. 1976;114(1):115-122. doi:10.1164/arrd.1976.114.1.115.

Funding: The authors received no funding for the current study.

Declaration of Conflicting Interests: Greg Malham: Globus (consultant), Device Technologies (travel), LifeHeathcare (travel). Nicholas Johnson: None. Matthew Claydon: Globus (consultant).

Ethics Approval: This study had institutional ethics and research approval.

Corresponding Author: Gregory M. Malham, Epworth Hospital, Melbourne, VIC 3121, Australia; gmalham@bigpond.net.au

Published 24 December 2021

This manuscript is generously published free of charge by ISASS, the International Society for the Advancement of Spine Surgery. Copyright (C) 2022 ISASS. To see more or order reprints or permissions, see http:// ijssurgery.com. 